\title{
Hemophilia: Where are we today?
}

1. Explain the pathogenesis and genetic mechanisms associated with hemophilias A and B.

2. Provide a historical account of hemophilia treatment and current available treatment options.

3. Describe laboratory assays available in the clinical laboratory used to diagnose patients with hemophilia and to monitor therapy.

\begin{abstract}
Hemophilia is a rare congenital bleeding disorder characterized by decreased levels of coagulation factor VIII or factor IX in the circulation. The disorder is associated with lifelong bleeding that ranges from mild to severe in affected individuals. Treatment requires replacement of the missing factor and is often associated with the development of antibodies that may exacerbate the bleeding diathesis seen in these individuals. The clinical laboratory plays a unique role in the diagnosis of hemophilia and in monitoring the effectiveness of standard replacement therapy, as well as many of the newer replacement therapies that have been developed.
\end{abstract}

Abbreviations: CSA - chromogenic substrate assay, FIX - factor IX, FVIII - factor VIII, HA hemophilia A, HB - hemophilia B, OSA - one stage assays

Index Term: acquired bleeding disorders, bleeding disorders, Christmas disease, congenital bleeding disorders, hemophilia, hemophilia A, hemophilia B, FVIII deficiency, FIX deficiency 
This edition of Focus consists of three articles that will 1) present a thorough review of hemophilia; 2) explain both current and new treatment options; and 3) describe the preanalytical, analytical and post-analytical variables associated with clinical assays used to diagnose and monitor treatment in patients with hemophilia.

Hemophilia is a rare bleeding disorder that involves a mutation in the genes for factor VIII (FVIII) or factor IX (FIX) resulting in decreased production of these clotting factors. ${ }^{1}$

Hemophilia A (HA) results from a deficiency of FVIII and hemophilia B (HB) results from a deficiency of FIX. The prevalence of HA and HB in the population is 1 in 5,000 and 1 in 30,000 live male births, respectively. ${ }^{1}$ Bleeding symptoms range from mild to severe and may manifest in hemarthrosis, soft-tissue bleeds, arthropathy, joint contractures, and disability. ${ }^{1}$ Congenital hemophilia A and B are sex-linked disorders inherited in a recessive pattern. Additionally, up to $30 \%$ of cases may arise de novo resulting from a spontaneous mutation. ${ }^{1}$ Hemophilia will discuss the pathogenesis and genetic mechanisms associated with hemophilia, as well as describe clinical and laboratory manifestations observed in patients with hemophilia.

The main treatment for hemophilia has been replacement therapy involving the administration of the deficient coagulation factor to achieve adequate hemostasis. ${ }^{2}$ Treatment is either on demand or given prophylactically. The goal of treatment is to prevent joint bleeds that can ultimately lead to disability. Much progress has been made in hemophilia treatment over the years with the introduction of plasma-derived and recombinant products. Because of bioengineering technology, scientists have been able to increase the half-lives of recombinant products resulting in few injections and less immunogenicity developing in treated patients. ${ }^{2}$ A number of gene therapy trials have also been conducted, and very promising results have been obtained suggesting a possible cure for hemophilia. ${ }^{3}$ Past, Present and Future Options in the 
Treatment of Hemophilia A will provide a historical account of the treatment for hemophilia A, as well as current available treatment options.

The clinical laboratory plays a critical role in diagnosing patients with hemophilia and in therapeutic monitoring. The diagnosis of hemophila can be established with factor assays for FVIII and FIX. The one-stage (clotting) assay (OSA) is the most widely-used assay for factor assay determination. ${ }^{4}$ It measures the ability of patient plasma to reduce the APTT clotting time of FVIII or FIX deficient plasma. In addition, a two-stage chromogenic substrate assay (CSA) may be used as an alternative. ${ }^{4}$ The first stage involves the generation of activated FX (FXa), and the second stage is involved in measuring the amount of generated FXa which is proportional to the amount of FVIII or FIX present in the patient plasma. ${ }^{5}$ Performance characteristics along with the advantages and disadvantages of these assays will be discussed in detail in Laboratory Monitoring of Factor VIII and FIX in Hemophilia Patients in this Focus series.

\section{Bibliography}

1. Mannucci PM, Tuddenham EGD. The Hemophilias - From Royal Genes to Gene Therapy. N Engl J Med 2001;344:1773-1779.

2. Peyvandi, F, Garagiola, I, Young, G. The Past and Future of Haemophilia: Diagnosis, Treatments, and its Complications. Lancet 2016;388:187-97.

3. Franchini, M, Mannucci, PM. The History of Hemophilia. Semin Thromb Hemost 2014;40:571-576.

4. Potgieter JJ, Damgaard M, Hillarp A. One-stage vs. Chromogenic Assays in Haemophilia A. Eur J Haematol 2015;94 Suppl 77:38-44. 
5. Peyvandi, F, Oldenburg, J, Friedmann, KD. A Critical Appraisal of One-state and Chromogenic Assays of Factor VIII Activity. J Thromb Haemost 2016;14:248-61. 